\title{
One-step staining of Neisseria gonorrhoeae in urethral discharge by methyl green-pyronin
}

\author{
N F HANNA,* D TAYLOR-ROBINSON, ${ }^{*} \mathrm{G}$ W CSONKA, $+\mathrm{J} R$ W HARRIS, + AND \\ I A AL-SOWAYGH‡ \\ From the *Division of Communicable Diseases, Clinical Research Centre, Harrow, Middlesex, and the \\ tPraed Street Clinic, St Mary's Hospital, London; and the $\neq$ University of Riyad, Saudi Arabia
}

SUMMARY Methyl green-pyronin (MGP) was used in a one-step procedure to stain smears of urethral discharge from 169 men. Duplicate smears were stained by Gram's method and discharge was cultured for Neisseria gonorrhoeae. The organisms were isolated from 67 specimens and intracellular diplococci were seen in 74 smears after Gram staining and in 77 after staining by MGP. Furthermore, more extracellular and intracellular diplococci were seen in smears stained by MGP than by Gram's method and the proportion of polymorphonuclear leucocytes found to contain the organisms was greater after staining with MGP. Staining with MGP is simple, rapid, inexpensive, and easily automated.

\section{Introduction}

The Gram stain is used routinely to stain urethral smears and requires five steps. It is well known that Neisseria gonorrhoeae organisms appear as red diplococci and that the nuclei of polymorphonuclear (PMiN) leucocytes are also a shade of this colour. Sowter and $\mathrm{McGee}^{1}$ were easily able to see gonococci in Fallopian tube tissues by using a Gram-methylgreen-pyronin-light-green (Gram-MGPLG) stain because the organisms were stained scarlet red in contrast to the green-blue staining of the cytoplasm and the blue nucleus of the epithelial cells. We thought, therefore, that this stain, or a slight modification of it (methyl green-pyronin, MGP), might be useful in examining smears from men with urethral discharge. Furthermore, the MGP-staining procedure requires a single step only and it might be useful in private practice and be easily automated for use in busy venereal disease clinics. Accordingly, specimens from men with urethral discharge have been cultured for $N$ gonorrhoeae and smears have been stained by Gram's method and by MGP and the results compared.

Address for reprints: Dr D Taylor-Robinson, Division of Communicable Diseases, Clinical Research Centre, Watford Road, Harrow, Middlesex

Received for publication 21 November 1979

\section{Patients and methods}

URETHRAL SMEARS

One hundred and sixty-nine men presenting with urethral discharge were examined. They were not consecutive cases but were randomly selected on the basis of the presence of a purulent discharge. After a specimen of discharge had been taken from each patient for culture on Thayer-Martin medium, further specimens were collected with a bacteriological loop and spread on two glass slides. Each smear covered a $1-\mathrm{cm}^{2}$ area marked on the undersurface of the slide in ink with a rubber stamp. The first smear was stained conventionally with Gram stain and the second smear with MGP stain; for each alternate patient this procedure was reversed-that is, MGP was used first and Gram second.

METHYL GREEN-PYRONIN (MGP) STAIN

The MGP stain was prepared by dissolving $0.15 \mathrm{~g}$ of methyl green (CI 42585) and $0.5 \mathrm{~g}$ of pyronin (CI 45005 ) in $2.5 \mathrm{ml}$ of $96 \%$ ethyl alcohol; $20 \mathrm{ml}$ of glycerin and $100 \mathrm{ml}$ of a solution of crystalline phenol (Analar) $(0.5 \% \mathrm{w} / \mathrm{v}$ in distilled water) were then added. The solution was mixed with $100 \mathrm{ml}$ of chloroform and allowed to stand at room temperature overnight. The mixture separated into two phases and the top layer, which comprised the 
stain, was removed carefully to avoid contamination with the chloroform. The stain was returned to the bottle at the end of a working day and shaken. Each batch of stain was used for a period of six months. All dyes were obtained from Raymond A Lamb Ltd (6 Sunbeam Road, London NW10 6IL).

\section{FIXATION AND STAINING PROCEDURES}

Smears of urethral discharge on glass slides were fixed by heat before being Gram stained. Smears for MGP-staining were dried in air; these slides were then immersed for two minutes in MGP stain contained in a coplin jar, rinsed in tap water, and dried.

\section{READING OF SMEARS}

MGP-stained and Gram-stained smears were read by two experienced observers, who each used a separate microscope at $\times 1000$ magnification. One hundred smears were examined by one observer, 69 by the other, and a few smears by both. The reading of smears was limited to the $1-\mathrm{cm}^{2}$ area and all intracellular and extracellular organisms within this area were counted, as much time being taken as was required to do this.

\section{Results}

OVERALL QUANTITATIVE ASSESSMENT

Urethral specimens from 169 patients were examined. Preliminary assessment indicated that the results obtained by one observer were similar to those obtained by the other; the combined results are therefore presented.

$N$ gonorrhoeae was cultured from specimens taken from 67 patients whose Gram-stained and MGPstained urethral smears also contained intracellular pink diplococci (table). Of the 102 culture-negative specimens, seven had given a positive result by Gram

TABLE Isolation of $N$ gonorrhoeae from the urethral discharge of 169 men and the occurrence of Gram-stained or MGP-stained intracellular and extracellular diplococci in their urethral smears

\begin{tabular}{llc}
\hline & \multicolumn{2}{c}{ No of urethral specimens } \\
\cline { 2 - 3 } Positive results to staining by & Culture + & Culture- \\
\hline $\begin{array}{l}\text { Gram's method (negatively stained) } \\
\text { Intracellular diplococci }\end{array}$ & 67 & 7 \\
$\quad$ Extracellular diplococci & 67 & 2 \\
MGP & & \\
$\quad$ Intracellular diplococci & 67 & 10 \\
Extracellular diplococci & 67 & 6 \\
Both methods & 67 & 4 \\
Total & 67 & 102 \\
\hline
\end{tabular}

+ Positive - negative stain and 10 a positive result by the MGP stain. Only four of these specimens gave positive results by both staining techniques. All specimens which were culture-positive showed diplococci by one of the two staining methods.

\section{QUALITATIVE ASSESSMENT}

\section{PMN leucocytes containing diplococci}

The proportion of PMN leucocytes seen to contain pink diplococci was greater after the smears were MGP-stained than after they were Gram-stained (fig 1). In addition, the number of PMN leucocytes containing $>10$ diplococci was greater when the smears were MGP-stained than when they were Gramstained (fig 2). Indeed, the distribution of results was very similar to that shown in fig 1 .

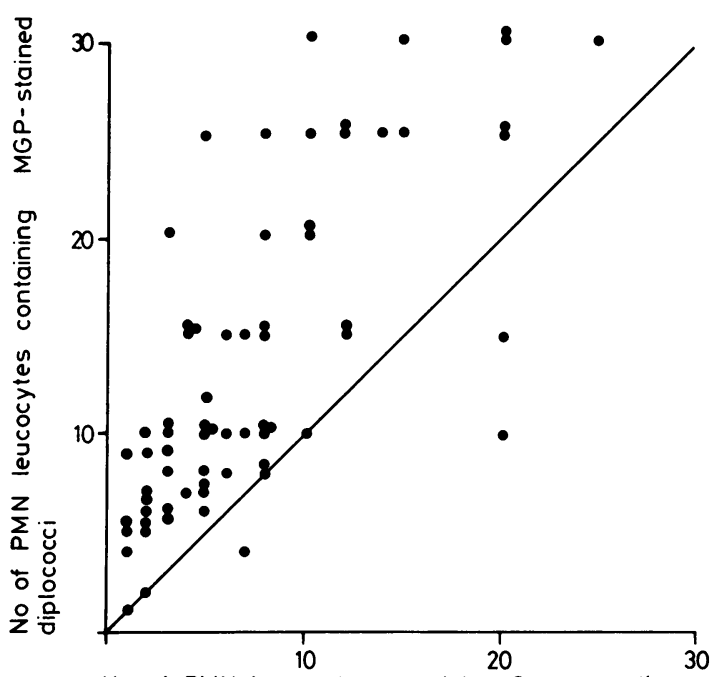

No of PMN leucocytes containing Gram-negative diplococci

FIG 1 Urethral smears from $67 \mathrm{~N}$ gonorrhoeae-culturepositive patients. The number (mean) of PMN leucocytes per high-power field $(\times 1000)$ of each smear containing diplococci that stained Gram-negatively are compared with the number containing diplococci that stained with MGP.

\section{Extracellular diplococci}

Extracellular, as well as intracellular, diplococci were seen in the urethral smears of all patients whose discharge was culture-positive, whether the smears were Gram-stained or MGP-stained (table). However, more extracellular diplococci were seen in MGP-stained smears than in Gram-stained smears (fig 2). In addition, a few of the culture-negative specimens containing intracellular diplococci also had extracellular organisms which stained by one of 


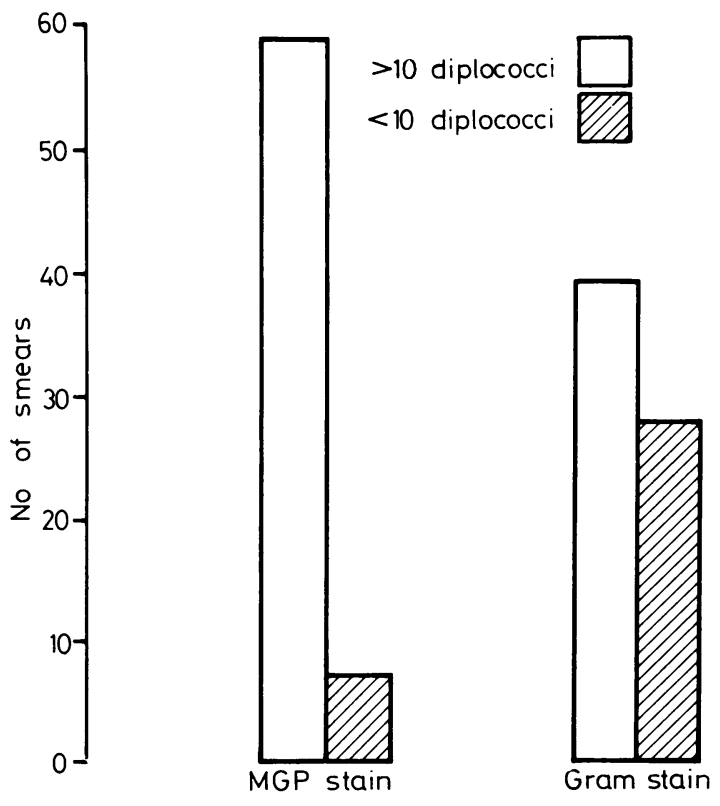

FIG 2 Urethral smears from 67 N gonorrhoeae-culturepositive patients. Number of smears containing $>10$ and $<10$ extracellular diplococci after staining with MGP and Gram's method.

the two methods (table). Five culture-negative specimens containing Gram-negative extracellular organisms only and four containing MGP-stained extracellular organisms only are not shown.

\section{Discussion}

Methyl green-pyronin stains gonococci red and the nuclei of the cells blue. It has, therefore, a greater differentiating capacity than methylene blue, which does not stain organisms or cell components differently. Furthermore, it is easier to detect gonococci within a cell using the MGP stain than it is using the Gram stain where organisms, particularly those overlying the nucleus, may not be seen. This may be the reason why more diplococci were seen within cells after staining with MGP. It is, however, difficult to explain why larger numbers of extracellular diplococci were seen after MGP-staining than after Gramstaining. It is possible only to speculate that organisms take up the MGP stain more avidly, and are therefore more easily seen, or that inadequate decolorisation in the Gram-stain procedure results in some organisms appearing Gram-positive.
We have found that methanol or formol-saline are superior to other fixatives because they increase the clarity of cell components. However, in this study, heat and air fixation provided sufficient clarity and were simple to perform.

The only problem using the MGP stain, as with methylene blue, is that it differentiates poorly between Gram-positive and Gram-negative organisms. Although organisms which are strongly Gram-positive may appear magenta colour with the MGP stain, while Gram-negative organisms appear red, the unwary observer may occasionally mistake a Gram-positive organism for a gonococcus. This may account for some of the "false-positive" results recorded for a few of the specimens in our series-that is, those where MGP stained intracellular organisms in a smear but gonococci were not isolated in culture. However, the number of such false-positive results produced by MGP-staining was only three more than by Gram-staining. It may be that these were due to the presence of commensal neisseriae or that the so-called false-positive results were not truly false but due to a relative failure to culture the organisms. A better assessment of the rate of false-positive results might be made by comparing the results of staining smears with Gram's method or MGP with those of staining duplicate smears with gonococcal fluorescent antibody, although differentiation between $N$ gonorrhoeae and $N$ meningitidis would still be difficult. Overall, we regard the problem of false-positive results with the MGP stain as a minor one, and it does not in any way outweigh the value of this staining procedure, which is both rapid and simple. In addition, the MGPstaining procedure is less costly than Gram staining because the components are cheaper and can be reused for a period of up to six months. MGP staining is also more easily automated since it involves only a single step.

We thank Mr C Sowter (Histopathology Department, Clinical Research Centre, Harrow) for advice on preparation and use of the MGP stain; the medical attendants and nursing staff of the Praed Street Clinic for their help; and the staff of the Department of Bacteriology, St Mary's Hospital Medical School, London, for culture facilities.

\section{Reference}

1. Sowter C, McGee ZA. Evaluation of a new technique for the demonstration of gonococci and other organisms in host cells. J Clin Path 1976; 29:433-7. 